\title{
Hall coefficient anomaly in the low-temperature high-field phase of $\mathrm{Sr}_{3} \mathrm{Ru}_{2} \mathrm{O}_{7}$
}

\author{
R. A. Borzi, ${ }^{1}$ A. McCollam, ${ }^{2}$ J. A. N. Bruin, ${ }^{3}$ R. S. Perry, ${ }^{4}$ A. P. Mackenzie, ${ }^{3}$ and S. A. Grigera ${ }^{3,5}$ \\ ${ }^{1}$ Instituto de Investigaciones Fisicoquímicas Teóricas y Aplicadas, and Departamento de Física, IFLP, Facultad de Ciencias Exactas, \\ UNLP-CONICET, 1900 La Plata, Argentina \\ ${ }^{2}$ Radboud University Nijmegen, High Field Magnet Laboratory, 6500 GL Nijmegen, The Netherlands \\ ${ }^{3}$ School of Physics and Astronomy, University of St Andrews, St Andrews KY16 9SS, United Kingdom \\ ${ }^{4}$ School of Physics, University of Edinburgh, Mayfield Road, Edinburgh EH9 3JZ, United Kingdom \\ ${ }^{5}$ Instituto de Física de Líquidos y Sistemas Biológicos, UNLP-CONICET, La Plata 1900, Argentina
}

(Received 18 July 2011; published 10 November 2011)

\begin{abstract}
We report a study of the Hall effect of high-purity $\mathrm{Sr}_{3} \mathrm{Ru}_{2} \mathrm{O}_{7}$ single crystals. We establish an empirical correlation between the onset of its unusual low-temperature, high-field phase and a pronounced dip in the fielddependent Hall coefficient. Unlike the order parameter obtained from measurements of anisotropic resistivity, which is affected by the formation of domains, the Hall effect feature seems to reflect the nature of the ordering within a single domain. We checked for violations of the Onsager relations for the off-diagonal components of the resistivity tensor but do not detect any. We compare our observations to those on materials that have long-wavelength spin structures, and discuss them in relation to a growing body of theoretical work on the nature of the low-temperature phase in $\mathrm{Sr}_{3} \mathrm{Ru}_{2} \mathrm{O}_{7}$.
\end{abstract}

DOI: 10.1103/PhysRevB.84.205112

PACS number(s): 71.18.+y, 71.27.+a, 74.70.Pq

The subject of this paper, $\mathrm{Sr}_{3} \mathrm{Ru}_{2} \mathrm{O}_{7}$, is the $n=2$ member of the Ruddlesden-Popper series of layered perovskites $\mathrm{Sr}_{n+1} \mathrm{Ru}_{n} \mathrm{O}_{3 n+1}$. It is a metal, with conduction primarily within its $\mathrm{RuO}_{2}$ planes, which stack as bilayers. In zero field it is a strongly enhanced paramagnet with a Wilson ratio of approximately $10 .{ }^{1}$ When grown to standard levels of purity, with mean free paths of a few hundred $\AA$, single crystals display metamagnetic transitions at fields ranging from $\approx 8 \mathrm{~T}$ (applied parallel to the crystallographic $c$ axis) to $5 \mathrm{~T}$ (applied in the crystallographic $a b$ plane), with evidence for metamagnetic quantum criticality for $B \| c .^{2,3}$ If the purity is enhanced, the approach to the critical point is cut off by the formation of a new phase, ${ }^{4}$ which displays twofold symmetric in-plane transport when subject to a small in-plane field component. ${ }^{5}$ Recent thermodynamic measurements have established it to be an equilibrium phase, ${ }^{6}$ and it has been identified as a candidate electronic nematic. . $^{5,7}$

Although an extensive set of magnetoresistance data has been acquired, ${ }^{2-5,8}$ no study has been performed of the Hall voltage at high fields and low temperatures. In this paper we report a series of measurements aiming to fill that gap, and show that the low-temperature phase in $\mathrm{Sr}_{3} \mathrm{Ru}_{2} \mathrm{O}_{7}$ is associated with a large and surprising signal in the Hall effect. Since the discovery of the asymmetry of the resistivity tensor in $2007,{ }^{5}$ a number of theories have been proposed to account for the transport anisotropy. ${ }^{9-18}$ However, none of them attempts a direct evaluation of this phenomenological order parameter, which is complicated by the existence of domains. The Hall effect anomaly will therefore provide additional input to attempts to understand the novel phase and its order parameter.

The crystals used in this study were grown in St. Andrews using a floating zone technique, and have residual resistivities in the range $0.4-0.6 \mu \Omega \mathrm{cm}$. We cut two types of samples: needlelike to maximize the transport signal, and in the shape of octagons to measure transport in different directions with minimal geometrical bias. Transport measurements were performed using dilution refrigerators in St. Andrews and
Nijmegen. Throughout this paper, as in Ref. 5, we define the crystalline $a$ and $b$ axes to be those of the tetragonal unit cell, i.e., parallel to the $\mathrm{Ru}-\mathrm{O}-\mathrm{Ru}$ directions in the $\mathrm{RuO}_{2}$ plane.

When the magnetic field is applied precisely perpendicular to the $\mathrm{Sr}_{3} \mathrm{Ru}_{2} \mathrm{O}_{7} a b$ plane, the anomalous phase is bounded by first-order metamagnetic phase transitions. They coincide with sharp changes in resistivity such that the phase is characterized by a pronounced peak in the magnetoresistance. ${ }^{4,8}$ In Fig. 1(a) we show the magnetoresistance $\rho_{x x}$ of a needle-shaped crystal of $\mathrm{Sr}_{3} \mathrm{Ru}_{2} \mathrm{O}_{7}$ (mean free path $\approx 3000 \AA$ ), for fields applied within $3^{\circ}$ of the $c$ axis. We observe a very sharp feature near $8 \mathrm{~T}$, the resistivity at its peak almost doubling the value at its base. As the temperature is increased this peak broadens rapidly, until only crossovers are seen in the trace at $1.3 \mathrm{~K}$. As discussed in Refs. 4 and 5, the extra scattering leading to the peak probably results from domain formation due to the breaking of an intrinsic symmetry.

The corresponding Hall resistivity $\rho_{x y}$ is shown in Fig. 1(b). As expected, it is field-linear at low fields, with a gradient that is consistent with two previous reports of the weak-field Hall coefficient in $\mathrm{Sr}_{3} \mathrm{Ru}_{2} \mathrm{O}_{7}{ }^{19,20}$ At high fields the traces begin to show long-period quantum oscillations superimposed on a strongly rising background. The most striking feature is the sharp and pronounced dip at low temperatures that takes place in the field and temperature region where the anisotropic phase exists. As with $\rho_{x x}$ data, the sharpness fades away with increasing temperature.

To set this dip in $\rho_{x y}$ in context, we briefly introduce the phenomena that contribute to the Hall signal in a nearly magnetic metal like $\mathrm{Sr}_{3} \mathrm{Ru}_{2} \mathrm{O}_{7}$. Common to all metals, there is the orbital contribution due to the Lorentz force on the quasiparticles. In weak field the sign of its contribution to $R_{H}=\rho_{x y} / B$ can be estimated in a multiband quasi-two-dimensional material using the scheme introduced by Ong. ${ }^{21}$ At low temperatures, where the mean free path is independent of $\mathbf{k}, \rho_{x y}$ becomes a weighted sum of negative contributions from electron pockets and positive ones from hole pockets. ${ }^{22}$ Early discussions of 


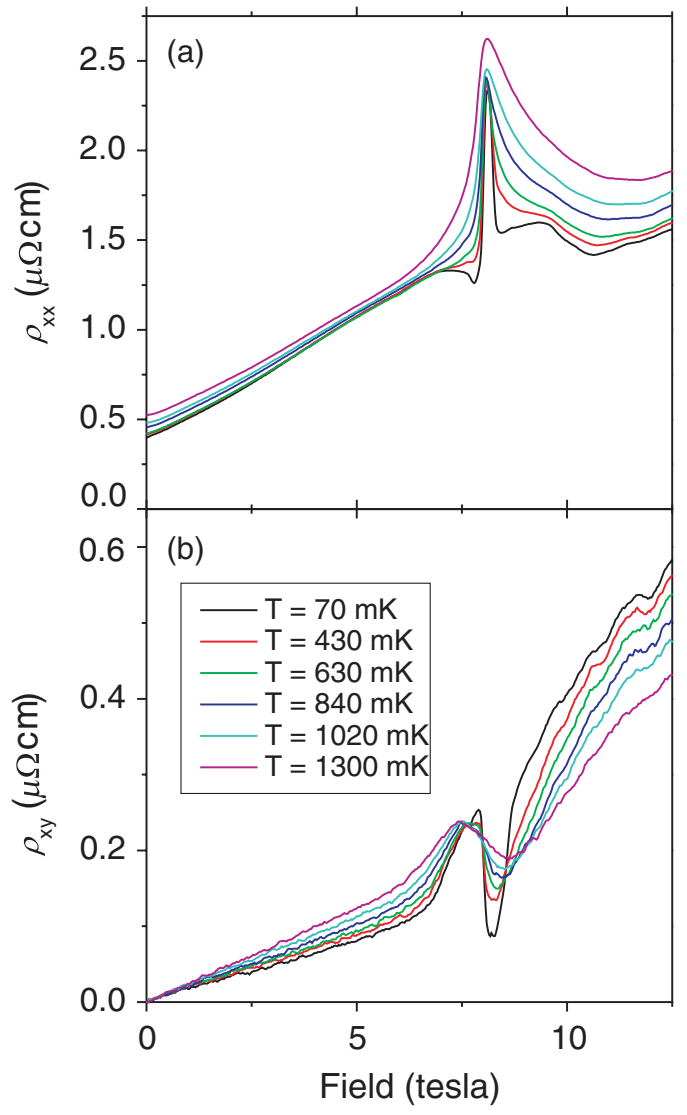

FIG. 1. (Color online) Magnetotransport of $\mathrm{Sr}_{3} \mathrm{Ru}_{2} \mathrm{O}_{7}$ for field applied within $3^{\circ}$ of the $c$ axis, measured in a needle-shaped sample. (a) Magnetoresistance as a function of field. At low temperatures a sharp peak develops within the novel phase. (b) Corresponding Hall resistivity. The linear term at low field is followed by a pronounced upturn, following the behavior of the magnetisation. At higher fields the traces show evidence of quantum oscillations. The most striking feature is the sharp and pronounced dip at low temperatures that takes place in the field and temperature region where the anisotropic phase exists.

the expected sign of the orbital contribution focused on the assumption that the Fermi surface would simply be a bilayer split version of the Fermi surface of tetragonal $\mathrm{Sr}_{2} \mathrm{RuO}_{4}$, resulting in the prediction of a negative Hall coefficient in contrast with observation. Now, however, it is known that this bilayer splitting is accompanied by gapping and zone folding due to the rotation of $\mathrm{RuO}_{2}$ octahedra. ${ }^{23,24}$ This results in large numbers of hole and electron pockets, and more uncertainty about the expected sign of the orbital contribution.

In addition to the orbital Hall effect, nearly magnetic materials also have an anomalous contribution to the Hall signal, described in early work by Karplus and Luttinger ${ }^{25}$ and extended and generalized by a number of authors over the past decade, invoking the concept of the Berry phase and Berry curvature. ${ }^{26-28}$ In any of these theories, the anomalous Hall coefficient is proportional to the magnetization $(M)$, and it adds to the orbital term to give the experimentally observed $R_{H}$. The anomalous Hall effect clearly makes a significant contribution to $R_{H}$ in $\mathrm{Sr}_{3} \mathrm{Ru}_{2} \mathrm{O}_{7}$. Both the qualitative temperature dependence of its weak-field Hall effect ${ }^{19,20}$ and the field-dependent data of Fig. 1(b) (outside the region of the anomalous dip) bear similarities to the overall field dependence of $M$ (see, e.g., Ref. 2). Although a quantitative account of these contributions would be desirable ${ }^{29}$ we found no reliable way of performing this analysis in our case. The scaling based on either Ref. 25, or Refs. 26-28 is not exact, and our overall conclusion is that the observed signal is a mix of orbital and anomalous contributions, similar in magnitude.

The main question that we address in this paper concerns the origin of the dip in $R_{H}$ shown in Fig. 1(b). There is no explanation for it in the theories of the anomalous Hall effect introduced above, because the magnetization rises in a series of steps through the transition region. One cannot rule out a peak in $\rho_{x x}$ being accompanied by a dip in $\rho_{x y}$ in the orbital Hall effect in a nearly compensated material with electron and hole pockets, but de Haas-van Alphen studies suggest that the Fermi surface changes on entering the phase are small, ${ }^{30}$ so this does not seem likely. Also, the strong temperature dependence shown in Fig. 1(b) and a strong purity dependence of the Hall signal argue against it being a standard Fermi-surface effect.

Instead, we investigate the intriguing possibility that the dip in the Hall coefficient is a manifestation of the microscopic nature of the phase itself. The connection between phase microscopics and Hall response has been stressed recently in relation to the helimagnet $\mathrm{MnSi}$, where a dip in the Hall coefficient under pressure is related to spin textures. ${ }^{29}$ The internal magnetoelectric symmetry of novel phases can also be of relevance, with a prediction that the Onsager relation $\sigma_{x y}=-\sigma_{y x}$ can be violated in some time-reversal symmetrybreaking states. ${ }^{31}$

As we have shown previously, within the novel phase, transport along otherwise symmetric directions becomes strongly anisotropic when an in-plane component of the applied magnetic field is introduced by tilting the sample. ${ }^{5}$ Small tilt angles appear to align domains, while for larger angles it is possible to define an order parameter using the observed anisotropy. The challenge for the present investigation was to work with samples that allowed an unbiased study of $\rho_{x x}, \rho_{y y}, \rho_{x y}$, and $\rho_{y x}$ for currents parallel, perpendicular, and at $45^{\circ}$ to the in-plane field component. As shown in Fig. 2(a), the simplest shape that allows this is an octagonal plate. ${ }^{33}$ If contacts are then placed at the center of each octagonal face, all the relevant transport coefficients can be measured on a sample whose shape is neutral. Using a fine wire saw, we cut four octagonal plates of typical dimension $1.5 \mathrm{~mm}$ across and $50 \mu \mathrm{m}$ thickness, with the $a$ and $b$ directions aligned with two principal axes of the octagons. Using these octagonal plates we measured all four components of the in-plane magnetoresistivity tensor.

Sample data from an octagonally cut crystal with a mean free path of $2000 \AA$ are shown in Figs. 2(b) and 2(c). The noise levels are higher than for the needle sample shown in Fig. 1, because the current densities in the octagons are much smaller and the need to reconfigure the contacts during the experiment precludes the use of low-temperature passive amplification. In Fig. 2(b) we show the evolution of the magnetoresistive signal at $100 \mathrm{mK}$ from fields applied close to the crystallographic $c$ axis to those tilted up to $33^{\circ}$ from it $\left(\theta \approx 57^{\circ}\right)$. In each case the tilt direction is along the $a$ axis, and the magnetoresistivity is 

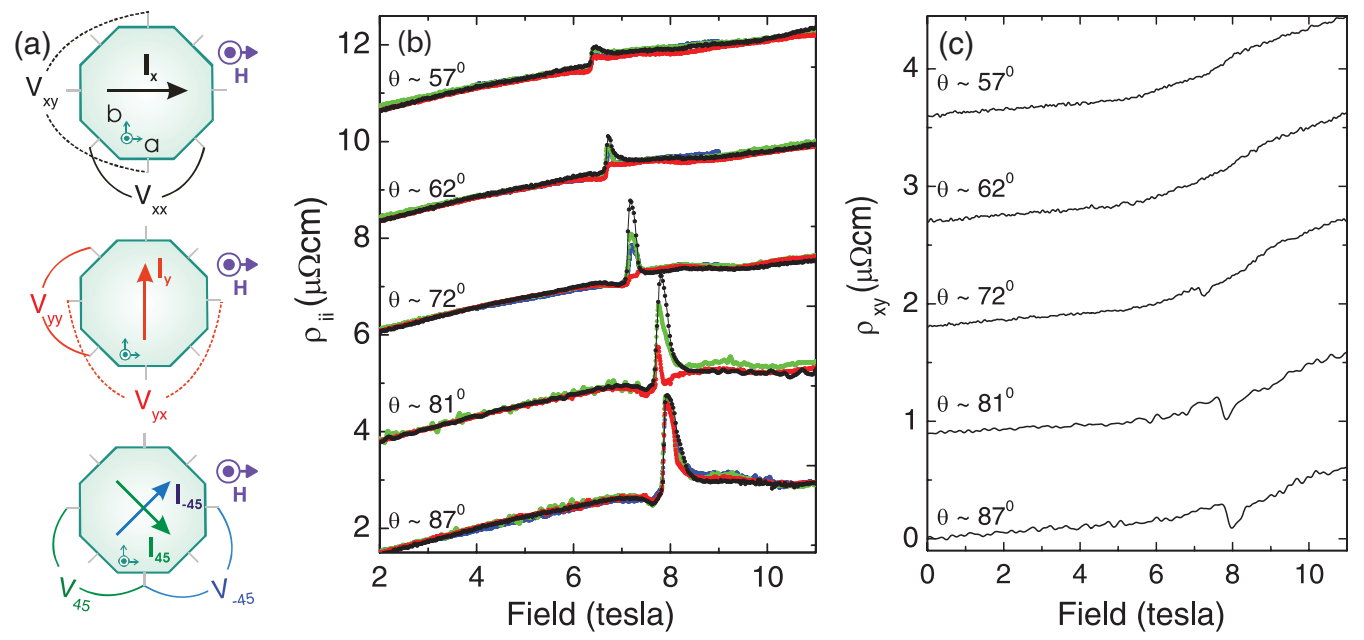

FIG. 2. (Color online) Magnetotransport at $100 \mathrm{mK}$ measured on octagonal single-crystal plates at different field angles $\theta$. The field is always perpendicular to the $b$ axis, and $\theta$ is measured from the $a$ axis. (a) Schematic view of the four different transport configurations we used to measure Hall effect and magnetoresistance for different combinations of in-plane current-field angle $\phi$ (the color of the arrows in the octagons correlates with the color of the traces in the other panels). The octagonal shape minimizes the extrinsic anisotropy when commuting the current and voltage contacts. (b) Near the $c$ axis $\left(\theta=90^{\circ}\right)$ and above $\approx 60^{\circ}$ the resistivity $\left(\rho_{i i}\right.$, with $i=x, y$,or \pm 45$)$ is quite similar along the four directions explored, in a wide field range (Ref. 32). However, within this angular range, there is a narrow field region where the resistivity in configurations $I_{x}$ (black) and $I_{y}$ (red) differs markedly. This defines the hard and easy directions, respectively, within the nematic phase. The resistivity along the remaining configurations $\left(I_{ \pm 45}\right.$, green and blue) is approximately the average of the hard and easy resistivities. (c) There is a sharp dip in the Hall signal $\rho_{x y}$ that coincides with the position where nematicity is observed; the feature fades away at low angles, where we measure little anisotropy in $\rho_{i i}$. The dip is most noticeable near the $c$ axis; here, the intrinsic anisotropy in the resistivity is suspected to be masked by domain formation. The traces at different angles have been shifted vertically for clarity.

shown measured with current flowing along the tilt direction (the "hard" axis, black trace, $\rho_{x x}$ ), perpendicular to it (the "easy" axis, red trace, $\rho_{y y}$ ), and at in-plane rotations $\phi$ of $45^{\circ}$.

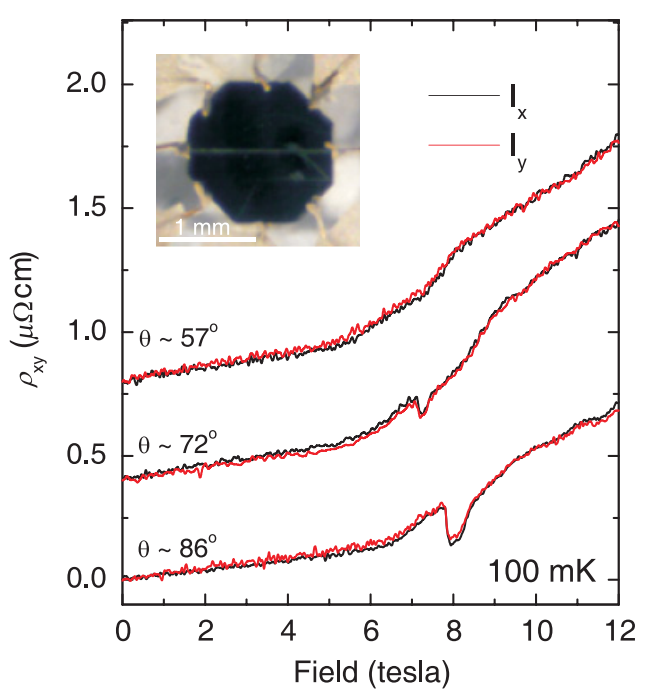

FIG. 3. (Color online) Comparison between $\left|\rho_{x y}\right|$ and $\left|\rho_{y x}\right|$, with current parallel $\left(I_{x}\right.$, black) and perpendicular $\left(I_{y}\right.$, red $)$ to the in-plane field, at different applied field angles $\theta$. The curves overlap within experimental error in both configurations at all fields, and for all angles explored. The Onsager relations are obeyed even at angles where the resistivity becomes highly anisotropic within the nematic phase (see the black and red resistivity curves in Panel a of Fig. 2). The traces at different angles have been shifted vertically for clarity. The inset shows a photograph of one of the samples, with eight gold wires attached with silver paint.
In line with our previous findings on needle samples, there is very little anisotropy for fields nearly parallel to $c$. At each value of $\theta$, the values of $\rho$ for $\phi= \pm 45^{\circ}$ are close to the geometrical mean of $\rho_{x x}$ and $\rho_{y y}$, confirming the accuracy of the octagonal shape and contact configuration of the sample.

The corresponding $\theta$ dependence of $\rho_{x y}$ is shown in Fig. 2(b). The dip in $\rho_{x y}$ tracks the features in $\rho_{x x}$, shrinking with decreasing $\theta$ and becoming invisible by $\theta \approx 62^{\circ}$. Even when there is a pronounced anisotropy between $\rho_{x x}$ and $\rho_{y y}$, we resolve no difference between $\rho_{x y}$ and $\rho_{y x}$ (see Fig. 3). The Onsager relation for the Hall conductivity is therefore obeyed in the new phase in $\mathrm{Sr}_{3} \mathrm{Ru}_{2} \mathrm{O}_{7}$.

Empirically, the data shown in Figs. 1-3 establish that the pronounced dip in $\rho_{x y}$, first seen at low temperatures for fields applied nearly parallel to $c$, correlates closely with the presence of the new phase as a function of temperature and tilt angle $\theta$. It also shares its strong purity dependence, disappearing in samples with mean free paths $<500 \AA$. It is tempting therefore to speculate that it is coupling somehow to the intrinsic order parameter of the phase. This is a significant observation, because it is believed ${ }^{5,30,34}$ that the formation of domains at high $\theta$ masks the macroscopic signature of this order parameter when observed in the magnetoresistance; the bulk anisotropy is close to zero when the domains are unaligned, and then rises as the in-plane field component is increased, becoming maximal for $70^{\circ}<\theta<80^{\circ}$ and disappearing for $\theta<60^{\circ}$. In contrast, an order parameter defined by the magnitude of the dip would fall monotonically with decreasing $\theta$, in line with our expectation for the $\theta$ dependence of the order parameter within a single domain. 
There are several possibilities for why this might occur. First, we note that the chiral spin structures proposed as the source of the Hall anomaly in MnSi (Ref. 29) are also present in some theories proposed for $\mathrm{Sr}_{3} \mathrm{Ru}_{2} \mathrm{O}_{7}$. ${ }^{14,35}$ Physically, they change the Hall response because the phase change experienced by electrons undergoing cyclic motion through this unusual spin background is equivalent to that felt orbiting in a magnetic field. Although the direct dipole coupling to the spin texture is small, the effective field can be much larger. Recently, similar physics has been predicted in response to strain fields in graphene, and Landau quantization with effective fields of hundreds of tesla has been observed. ${ }^{36}$ In $\mathrm{MnSi}$, the effective fields are a few tesla, and if they were present here, inspection of Fig. 2 suggests an effective internal field $B_{\text {int }}$ of 3-5 T, opposing the externally applied one $B_{\text {ext }}$. This effective field would have consequences for the spectrum of de Haas-van Alphen (dHvA) oscillations in $\mathrm{Sr}_{3} \mathrm{Ru}_{2} \mathrm{O}_{7}$. The field would not be spatially uniform, since it is linked to the spin textures, so dHvA frequencies would be broadened. They would also be higher than the true frequency by the ratio $B_{\text {ext }} /\left(B_{\text {ext }}-B_{\text {int }}\right)$. Experimentally, frequencies of approximately 2.3 and $1.0 \mathrm{kT}$ are observed within the anomalous phase. ${ }^{30}$ The restricted field width over which they are observed means that they are strongly broadened, and definite identification with frequencies seen at lower fields is complicated because of the strongly nonlinear $M(H)$ in the metamagnetic region. Although the most natural analysis identifies them with frequencies of 0.9 and $1.8 \mathrm{kT}$ seen outside the phase,${ }^{30}$ the data do not rule out an alternative interpretation associating them with 0.43 and $0.9 \mathrm{kT}$ frequencies from outside the phase, taking into account a significant reduction of the effective field within it. Further experiments are desirable to investigate that possibility.

In an alternative scenario that does not invoke spin structures and effective internal fields, the Hall response might be related to intrinsic electronic anisotropy. In $\mathrm{La}_{2-x-y} \mathrm{Nd}_{y} \mathrm{Sr}_{x} \mathrm{CuO}_{4}$, the onset of the formation of microscopic stripes identified in scattering experiments was shown to coincide with a sharp fall in the Hall coefficient, even though microscopic twinning of the stripes precluded the observation of any macroscopic resistive anisotropy. ${ }^{37} \mathrm{~A}$ third possibility to be borne in mind for future work is that the Hall effect anomaly that is seen at current levels of purity is the precursor of an even stronger dip toward zero in higher purity samples that might signal the onset of a topologically protected state of some kind. For the moment, all these interpretations are speculative, but motivate further investigation of the unusual high-field phase in $\mathrm{Sr}_{3} \mathrm{Ru}_{2} \mathrm{O}_{7}$.

In conclusion, we have studied the field-dependent Hall coefficient in high-purity single crystals of $\mathrm{Sr}_{3} \mathrm{Ru}_{2} \mathrm{O}_{7}$, establishing an empirical correlation between the onset of an unusual low-temperature, high-field phase and a pronounced dip in the field-dependent Hall coefficient.

We thank the UK Royal Society, EPSRC, ANPCYT, and CONICET (Argentina) for valuable financial support, and A. G. Green for useful discussions.
${ }^{1}$ S.-I. Ikeda, Y. Maeno, S. Nakatsuji, M. Kosaka, and Y. Uwatoko, Phys. Rev. B 62, R6089 (2000).

${ }^{2}$ R. S. Perry, L. M. Galvin, S. A. Grigera, L. Capogna, A. J. Schofield, A. P. Mackenzie, M. Chiao, S. R. Julian, S. I. Ikeda, S. Nakatsuji, Y. Maeno, and C. Pfleiderer, Phys. Rev. Lett. 86, 2661 (2001).

${ }^{3}$ S. A. Grigera, R. S. Perry, A. J. Schofield, M. Chiao, S. R. Julian, G. G. Lonzarich, S. I. Ikeda, Y. Maeno, A. J. Millis, and A. P. Mackenzie, Science 294, 329 (2001).

${ }^{4}$ S. A. Grigera, P. Gegenwart, R. A. Borzi, F. Weickert, A. J. Schofield, R. S. Perry, T. Tayama, T. Sakakibara, Y. Maeno, A. G. Green, and A. P. Mackenzie, Science 306, 1155 (2004).

${ }^{5}$ R. A. Borzi, S. A. Grigera, J. Farrell, R. S. Perry, S. J. S. Lister, S. L. Lee, D. A. Tennant, Y. Maeno, and A. P. Mackenzie, Science 315, 214 (2007).

${ }^{6}$ A. W. Rost, R. S. Perry, J.-F. Mercure, A. P. Mackenzie, and S. A. Grigera, Science 325, 1360 (2009).

${ }^{7}$ E. Fradkin, S. A. Kivelson, M. J. Lawler, J. P. Eisenstein, and A. P. Mackenzie, Annu. Rev. Condens. Matter Phys. 1, 153 (2010).

${ }^{8}$ R. S. Perry, K. Kitagawa, S. A. Grigera, R. A. Borzi, A. P. Mackenzie, K. Ishida, and Y. Maeno, Phys. Rev. Lett. 92, 166602 (2004).

${ }^{9}$ H.-Y. Kee and Y. B. Kim, Phys. Rev. B 71, 184402 (2005).

${ }^{10}$ B. Binz, H. B. Braun, T. M. Rice, and M. Sigrist, Phys. Rev. Lett. 96, 196406 (2006).

${ }^{11}$ H. Yamase and P. Jakubczyk, Phys. Rev. B 82, 155119 (2010).

${ }^{12}$ M. H. Fischer and M. Sigrist, Phys. Rev. B 81, 064435 (2010).
${ }^{13}$ W.-C. Lee and C. Wu, Phys. Rev. B 80, 104438 (2009).

${ }^{14}$ A. M. Berridge, A. G. Green, S. A. Grigera, and B. D. Simons, Phys. Rev. Lett. 102, 136404 (2009).

${ }^{15}$ S. Raghu, A. Paramekanti, E.-A. Kim, R. A. Borzi, S. A. Grigera, A. P. Mackenzie, and S. A. Kivelson, Phys. Rev. B 79, 214402 (2009).

${ }^{16}$ C. M. Puetter, J. G. Rau, and H. Y. Kee, Phys. Rev. B 81, 081105(R) (2010).

${ }^{17}$ H. Yamase, Phys. Rev. B 76, 155117 (2007).

${ }^{18}$ H. Yamase and A. A. Katanin, J. Phys. Soc. Jpn. 76, 073706 (2007).

${ }^{19}$ R. S. Perry, L. M. Galvin, A. P. Mackenzie, D. M. Forsythe, S. R. Julian, S. I. Ikeda, and Y. Maeno, Physica B 284-288, 1469 (2000).

${ }^{20}$ Y. Liu, R. Jin, Z. Q. Mao, K. D. Nelson, M. K. Haas, and R. J. Cava, Phys. Rev. B 63, 174435 (2001).

${ }^{21}$ N. P. Ong, Phys. Rev. B 43, 193 (1991).

${ }^{22}$ E.g., A. P. Mackenzie, S. R. Julian, A. J. Diver, G. G. Lonzarich, N. E. Hussey, Y. Maeno, S. Nishizaki, and T. Fujita, Physica C 26, 510 (1996).

${ }^{23}$ D. J. Singh and I. I. Mazin, Phys. Rev. B 63, 165101 (2001).

${ }^{24}$ A. Tamai et al., Phys. Rev. Lett. 101, 026407 (2008).

${ }^{25}$ R. Karplus and J. M. Luttinger, Phys. Rev. 95, 1154 (1954).

${ }^{26}$ G. Sundaram and Q. Niu, Phys. Rev. B 59, 14915 (1999).

${ }^{27}$ M. Onoda and N. Nagaosa, J. Phys. Soc. Jpn. 71, 19 (2002).

${ }^{28}$ T. Jungwirth, Q. Niu, and A. H. MacDonald, Phys. Rev. Lett. 88, 207208 (2002). 
${ }^{29}$ M. Lee, W. Kang, Y. Onose, Y. Tokura, and N. P. Ong, Phys. Rev. Lett. 102, 186601 (2009).

${ }^{30}$ J.-F. Mercure, S. K. Goh, E. C. T. O'Farrell, R. S. Perry, M. L. Sutherland, A. W. Rost, S. A. Grigera, R. A. Borzi, P. Gegenwart, and A. P. Mackenzie, Phys. Rev. Lett. 103, 176401 (2009).

${ }^{31}$ C. M. Varma, V. M. Yakovenko, and A. Kapitulnik, e-print arXiv:1007.1215 (to be published).

${ }^{32}$ The geometrical factor to convert between voltage and resistivity for the $I_{ \pm 45}$ configuration was fixed by making the value of the resistivity at low fields coincide with $\rho$ along the principal axes in the case of field applied along the $c$ axis, and was kept constant for all angles.
${ }^{33}$ Having shape-neutral samples is particularly important if domains might be present since sample shape might affect their configuration.

${ }^{34}$ C. Stingl, R. S. Perry, Y. Maeno, and P. Gegenwart, Phys. Rev. Lett. 107, 026404 (2011).

${ }^{35}$ A. M. Berridge, S. A. Grigera, B. D. Simons, and A. G. Green, Phys. Rev. B 81, 054429 (2010).

${ }^{36}$ N. Levy, S. A. Burke, K. L. Meaker, M. Panlasigui, A. Zettl, F. Guinea, A. H. Castro Neto, and M. F. Crommie, Science 329, 544 (2010).

${ }^{37}$ T. Noda, H. Eisaki, and S. Uchida, Science 286, 265 (1999). 